\title{
Paraspinal Myositis in Patients with COVID-19 Infection
}

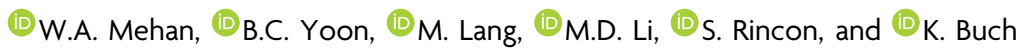

\begin{abstract}
SUMMARY: Myalgia is a previously reported symptom in patients with COVID-19 infection; however, the presence of paraspinal myositis has not been previously reported. We report MR imaging findings of the spine obtained in a cohort of 9 patients with COVID-19 infection who presented to our hospital between March 3, 2020 and May 6, 2020. We found that 7 of 9 COVID-19 patients (78\%) who underwent MR imaging of the spine had MR imaging evidence of paraspinal myositis, characterized by intramuscular edema and/or enhancement. Five of these 7 patients had a prolonged hospital course (greater than 25 days). Our knowledge of the imaging manifestations of COVID-19 infection is expanding. It is important for clinicians>a to be aware of the relatively high frequency of paraspinal myositis in this small cohort of patients with COVID-19 infection.
\end{abstract}

ABBREVIATIONS: COVID-19 = coronavirus disease 2019; SARS-CoV-2 = Severe Acute Respiratory Syndrome coronavirus 2

I: nformation regarding the imaging manifestations in patients infected with Severe Acute Respiratory Syndrome coronavirus 2 (SARS-CoV-2) has been rapidly evolving; however, most of the imaging studies have primarily focused on the pulmonary, gastrointestinal, and cardiac manifestations of coronavirus disease 2019 (COVID-19). ${ }^{1-15}$ Emerging case reports have described neuroimaging manifestations seen in patients with COVID-19 infection, including acute intracranial hemorrhage, demyelinating lesions, and encephalitis. ${ }^{16}$

The presence of paraspinal muscular pathology in the setting of COVID-19 infection has not been previously reported. Although the most common symptoms of COVID-19 infection include cough, shortness of breath, and fever, myalgia has been reported in up to $50 \%$ of patients with the infection. ${ }^{17-18}$ The purpose of this study was to describe the imaging findings of suspected myositis in a small cohort of patients with COVID-19 infection undergoing MR imaging of the spine.

Received May 26, 2020; accepted after revision June 12.

From the Department of Radiology, Massachusetts General Hospital, Boston, Massachusetts.

Please address correspondence to Karen Buch, MD, Department of Radiology, Massachusetts General Hospital, 55 Fruit St, Boston, MA 02118, United States; e-mail: kbuch@partners.org; @MGHNeuroRad; @KBuchRad

- Indicates open access to non-subscribers at www.ajnr.org

Indicates article with supplemental on-line table.

http://dx.doi.org/10.3174/ajnr.A6711

\section{CASE SERIES}

This study was an institutional review board-approved, retrospective study performed at a single large academic institution. Between March 3, 2020 and May 6, 2020, 641 patients presented to our institution for medical care and tested positive for COVID-19 by reverse transcriptase polymerase chain reaction. A total of 9 patients with COVID-19 infection at our institution underwent MR imaging of the spine for evaluation of spinal pathology with indications including back pain, lower extremity weakness, and lower extremity paresthesia.

All imaging was performed on either a 1.5T (Signa HX and Signa Excite HDx, GE Healthcare; Avanto, Siemens) or 3T scanner (Discovery MR750, GE Healthcare; Tim Trio and Skyra, Siemens). All MR imaging examinations of the spine included sagittal T1-weighted imaging, sagittal non-fat-saturated T2weighted imaging, sagittal fat-saturated T2-weighted imaging, and axial T2-weighted imaging. Postcontrast sequences were performed in a subset of this cohort.

Evaluation of the MR imaging included an assessment for spinal cord signal abnormality and clumping or thickening of the cauda equina nerve roots, bone marrow edema suggestive of an acute process, edema and/or enhancement within the intervertebral disks, and edema and/or enhancement within the paraspinal musculature. Myositis was defined as intramuscular edema manifested by $\mathrm{T} 2$ hyperintensity and/or enhancement within the paraspinal muscles which was present in the absence of, or disproportionate to the presence of minimal edema in the posterior subcutaneous soft tissues. The locations of these findings were 


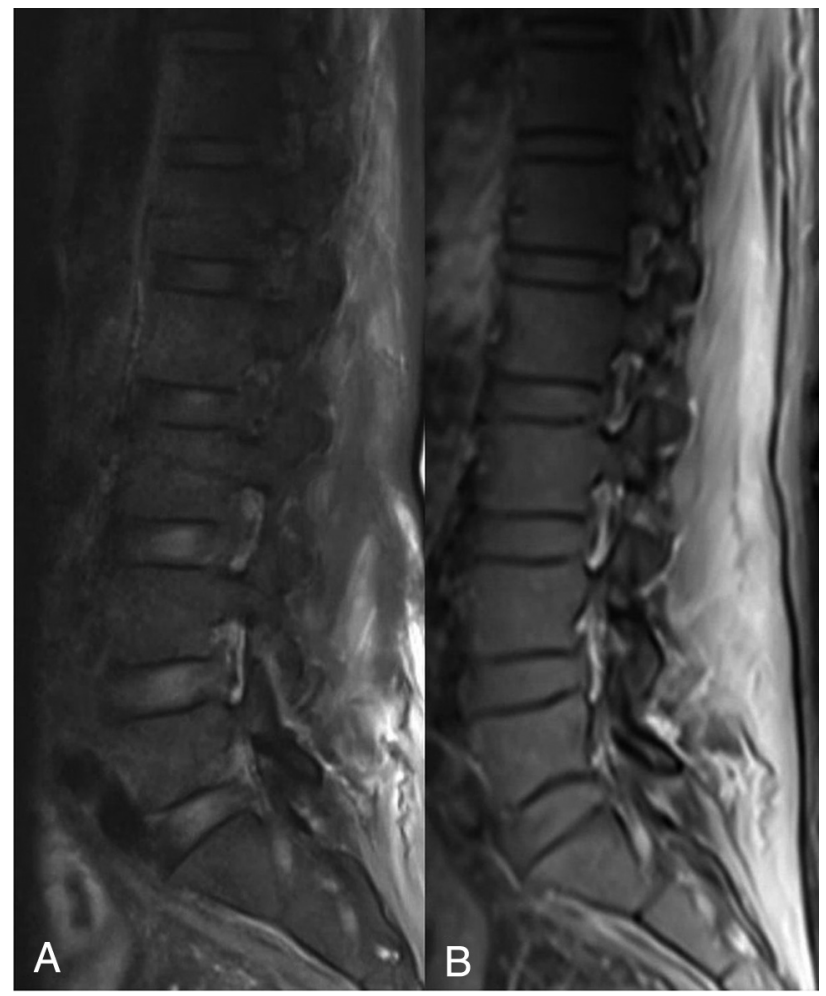

FIG 1. A 56-year-old man with COVID-19. A, sagittal fat-saturated T2-weighted image of the lumbar spine demonstrating increased T2 signal intensity within the posterior paraspinal muscles. $B$, postcontrast fat-saturated T1-weighted image of the lumbar spine demonstrating diffuse enhancement within the posterior paraspinal muscles.

recorded in reference to the vertebral body level for each patient. Indications for spine MR imaging included a report of back pain in 5 patients, bilateral leg pain in 2 patients, imbalance in 1 patient, and 1 patient who was found unconscious after a suicide attempt.

A complete cervical, thoracic, and lumbar spine MR imaging was performed in 3 patients, an isolated cervical spine MR imaging was performed in 1 patient, thoracolumbar MR imaging was performed in 3 patients, and isolated lumbar spine MR imaging was performed in 2 patients (On-line Table). Four of the $9 \mathrm{MR}$ imaging examinations were performed with contrast and the remaining 5 MRIs were performed without contrast.

Prior MR imaging of the spine was available for 3 patients in this cohort confirming that no findings of myositis were seen on prior spine MR imaging. Additionally, the electronic medical records were searched for each patient and confirmed that none of these patients had been previously treated for myositis. One patient in this cohort had a single-level laminectomy in the thoracic spine for treatment of an epidural abscess 5 years earlier. No history of prior spine surgery was present in the remaining 8 patients.

Imaging findings were correlated with clinical parameters. Patient age, sex, length of hospital stay, respiratory failure requiring intubation, presence of a superimposed bacterial infection, and serum inflammatory markers were recorded for each patient. The recorded serum inflammatory

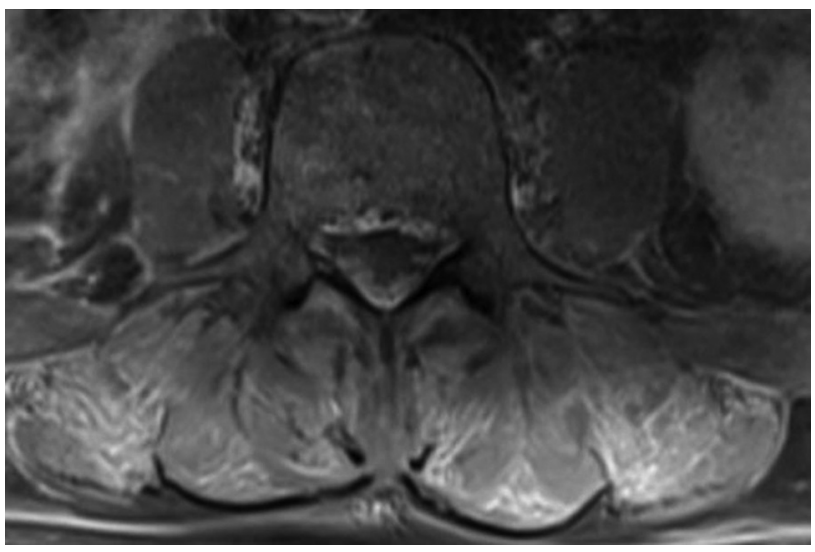

FIG 2. Same 56-year-old man with COVID-19 seen in Fig 1. Axial postcontrast, fat-saturated Tl-weighted image of the lumbar spine demonstrates symmetric areas of intramuscular enhancement involving the erector spinae and multifidus muscles bilaterally.

markers included creatine kinase levels (normal range 60$400 \mathrm{U} / \mathrm{L}$ ), erythrocyte sedimentation rate (normal range 0$20 \mathrm{~mm} / \mathrm{h}$ ), C-reactive protein (normal range $<8.0 \mathrm{mg} / \mathrm{L}$ ), creatinine level (normal range $0.6-1.5 \mathrm{mg} / \mathrm{dL}$ ), and D-dimer (normal range $<500 \mathrm{mg} / \mathrm{mL}$ ). All serum inflammatory markers recorded were performed within 12 hours of the MR imaging examination.

Of the 9 patients included in this cohort, the mean age was 55 years (standard deviation 17 years, range: $30-87$ ). Six of the 9 patients required admission to the hospital for treatment of COVID-19 infection, and 4 of these patients were intubated. The mean time between hospital admission and/or symptom onset and acquisition of the MR imaging scan was 17 days (range 1-35 days following admission). None of the patients in this cohort were diagnosed with having a superimposed bacterial infection over the duration of their hospital courses.

Seven of $9(78 \%)$ patients who underwent spine MR imaging demonstrated evidence of myositis on MR imaging with involvement of the erector spinae muscles and multifidus muscles (Figs 1-3). In all 7 patients with MR imaging findings of myositis, it occurred exclusively in the lumbar spine and involved multiple vertebral body levels (On-line Table). In all cases, the myositis was bilateral.

All patients with lumbar spine imaging ( 8 total) had trace to mild edema within the superficial subcutaneous soft tissues. In the 7 patients with lumbar spine MR imaging who had imaging findings suggestive of myositis, the edema in the paraspinal musculature was out of proportion to the degree of superficial subcutaneous edema.

None of the patients in this cohort demonstrated signal abnormality or enhancement within the visualized portion of the spinal cord or clumping or thickening of the cauda equina nerve roots. Abnormal edema and/or enhancement within the intervertebral disks was not seen in any patient in this cohort. Two of the 9 patients in this cohort had degenerative changes related to moderate facet hypertrophy confined to 2 levels within the lumbar spine. There was no evidence of reactive marrow edema in these patients related to degenerative disease. 


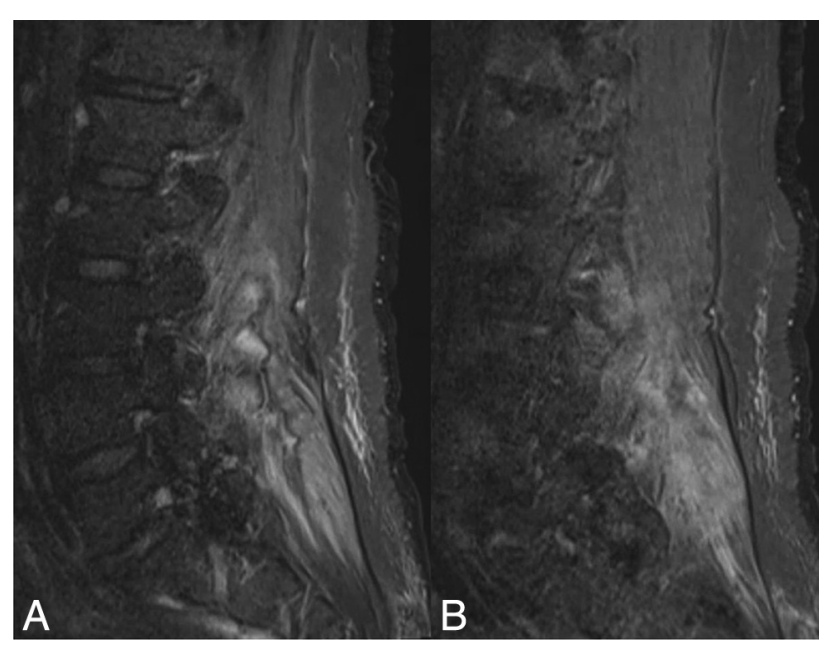

FIG 3. A 54-year-old woman with COVID-19. Sagittal fat-saturated T2-weighted imaging of the lumbar spine demonstrates nearly symmetric areas of increased T2 signal intensity within the posterior paraspinal muscles bilaterally. A, right parasagittal view of the T2 signal hyperintensity in the right paraspinal musculature. $B$, left parasagittal view of the T2 signal hyperintensity in the left paraspinal musculature.

One patient included in this cohort had a history of adrenal carcinoma with a known metastatic lesion at the L5 level, which was unchanged in appearance from multiple prior MR imaging examinations, and was not associated with new marrow edema or extension of disease.

Of the 7 patients with MR evidence of myositis, 5 were admitted to the hospital with a length of stay greater than 25 days, and 4 of these 5 patients were intubated over the course of their hospital admission. Three of the 7 patients with myositis demonstrated elevated serum inflammatory markers (as shown in the On-line Table). MR findings suggestive of myositis were seen in 2 patients who did not require hospital admission, did not have symptoms of respiratory failure, and did not have serum inflammatory markers collected.

None of the patients in this cohort received hydroxychloroquine or remdesivir for treatment of COVID-19 infection.

\section{DISCUSSION}

Of the small cohort of patients with COVID-19 infection who underwent MR imaging at our institution for evaluation of the spine, $78 \%$ (7 of 9) of patients had intramuscular edema and imaging findings suggestive of myositis, and all cases of myositis were found in the lumbar spine. These findings occurred in the absence of spine trauma. Three of the 7 patients in this cohort with MR imaging findings suggestive of myositis had elevated inflammatory markers. Two patients in our cohort did not demonstrate imaging manifestations of lumbar myositis; 1 had a relatively short hospital admission of 10 days, and the other patient was asymptomatic and did not require hospital admission. The patients in this cohort who had imaging of the cervical and thoracic spine demonstrated no MR imaging findings to suggest myositis in these segments of the spine.
Possible etiologies of the observed paraspinal myositis include direct muscular viral infection with SARS-CoV-2, an immunemediated, parainfectious inflammatory response given the elevated serum inflammatory markers in several patients, or drug-mediated effects. An additional consideration for these findings includes sequelae of a critical illness myopathy that has been previously described in up to $62 \%$ of intensive care unit patients who failed to be wean from the ventilator. ${ }^{19-20}$ While it is possible that these findings could be compounded by third spacing with gravitationally dependent edema, this is felt to be less likely as edema and/or paraspinal muscular enhancement was found to be out of proportion to the degree of overlying superficial subcutaneous edema, which was found to be trace to mild in all patients. Of the 7 patients with MR imaging findings of myositis, 3 patients were never intubated, and 4 patients had MR imaging findings suggestive of myositis with MR imaging performed within 10 days of admission. These findings suggest that patients may have been relatively early in their COVID-19 infection course and these findings may be unrelated to a critical illness myopathy. Viral myositis is a known entity reportedly caused by several other viruses including influenza, HIV, hepatitis C, and Middle East Respiratory Syndrome Coronavirus. ${ }^{21-25}$ The results of this study are consistent with Beydon et al ${ }^{19}$ who demonstrated MR features of myositis involving the pelvis and thighs in a patient with COVID-19 infection.

This is a small series of patients, in part related to the low frequency of back pain complaints in patients with COVID-19 infection. Furthermore, severe manifestations of COVID-19 may render patients too unstable or unable to undergo MR imaging of the spine. There are numerous confounders in these patients including heterogeneous sampling of serum inflammatory markers, lack of clear information regarding how long patients had the infection before experiencing symptoms of back pain and weakness, and heterogeneous treatment algorithms. These variations in practice patterns, documentation, and recorded histories make it difficult to determine dominant etiologies contributing to these imaging manifestations.

In conclusion, the findings of this study demonstrate a high frequency of myositis in patients with COVID-19 infection reporting myalgia in a small series. It is important for health care providers and radiologists to be aware of these clinical features and imaging findings.

Disclosures: William A. Mehan-UNRELATED: Consultancy: Kura Oncology, Comments: Independent reviewer of head and neck imaging studies for a clinical trial; Expert testimony: CRICO and other health insurance companies, Comments: Expert opinion for medicolegal cases involving neuroimaging.

\section{REFERENCES}

1. Zeng Z, Xu L, Xie XY, et al. Pulmonary pathology of early phase COVID-19 pneumonia in a patient with a benign lung lesion. Histopathology 2020 May 6. [Epub ahead of print] CrossRef Medline

2. Jafari R, Cegolon L, Jafari A, et al. Large saddle pulmonary embolism in a woman infected by COVID-19 pneumonia. Eur Heart $J$ 2020;41:2133 CrossRef Medline

3. Ji M, Yuan L, Shen W, et al. Characteristics of disease progress in patients with coronavirus disease 2019 in Wuhan, China. Epidemiol Infect 2020;148:e94 CrossRef Medline 
4. Yu Q, Wang Y, Huang S, et al. Multicenter cohort study demonstrates more consolidation in upper lungs on initial CT increases the risk of adverse clinical outcome in COVID-19 patients. Theranostics 2020;10:5641-48 CrossRef

5. Wu J, Pan J, Teng D, et al. Interpretation of CT signs of 2019 novel coronavirus (COVID-19) pneumonia. Eur Radiology 2020 May 4. [Epub ahead of print] CrossRef Medline

6. Pozzessere C, Rotzinger DC, Ghaye B, et al. Incidentally discovered COVID-19 pneumonia: the role of diagnostic imaging. Eur Radiology 2020 May 4. [Epub ahead of print] CrossRef Medline

7. Liya G, Yuguang W, Jian L, et al. Studies on viral pneumonia related to novel coronavirus SARS-CoV-2, SARS-CoV, and MERS-CoV: a literature review. APMIS 2020 May 4. [Epub ahead of print] CrossRef Medline

8. Guneyli S, Atceken Z, Dogan $\mathrm{H}$, et al. Radiological approach to COVID-19 pneumonia with an emphasis on chest CT. Diagn Interv Radiology 2020;26:323-32 CrossRef

9. Ding X, Xu J, Zhou J, et al. Chest CT findings of COVID-19 pneumonia by duration of symptoms. Eur J Radiology 2020;127:109009 CrossRef

10. Middeldorp S, Coppens M, van Haaps TF, et al. Incidence of venous thromboembolism in hospitalized patients with COVID-19. J Thromb Haemost 2020 May 5. [Epub ahead of print] CrossRef Medline

11. Scialpi M, Scialp S, Piscioli I, et al. Pulmonary thromboembolism in critically ill COVID-19 patients. Int J Infect Dis 2020;95:361-62 CrossRef Medline

12. Leonard-Lorant I, Delabranche X, Severac F, et al. Acute pulmonary embolism in COVID-19 patients on CT angiography and relationship to D-dimer levels. Radiology 2020 Apr 23. [Epub ahead of print] CrossRef Medline

13. Grillet F, Behr J, Calame P, et al. Acute pulmonary embolism associated with COVID-19 pneumonia detected by pulmonary CT angiography. Radiology 2020 Apr 23. [Epub ahead of print] CrossRef Medline
14. Babapoor-Farrokhran S, Gill D, Walker J, et al. Myocardial injury and COVID-19: possible mechanisms. Life Sci 2020;253:117723 CrossRef Medline

15. Liu K, Pan M, Xiao Z, et al. Neurological manifestations of the coronavirus (SARS-CoV-2) pandemic 2019-2020. J Neurol Neurosurg Psychiatry 2020;91:669-70 CrossRef Medline

16. Zanin L, Saraceno G, Panciani PP, et al. SARS-CoV-2 can induce brain and spine demyelinating lesions. Acta Neurochir (Wien) 2020;162:1491-94 CrossRef Medline

17. Chen $\mathrm{Y}, \mathrm{Zhao} \mathrm{M}, \mathrm{Wu} \mathrm{Y}$, et al. Epidemiological analysis of the early 38 fatalities in Hubei, China, of the coronavirus disease 2019. J Glob Health 2020;10:011004 CrossRef Medline

18. Beydon M, Chevalier $\mathrm{K}, \mathrm{Al}$ Tabaa $\mathrm{O}$, et al. Myositis as a manifestation of SARS-CoV-2. Ann Rheum Dis 2020 CrossRef

19. Latronico N, Bolton CF. Critical illness polyneuropathy and myopathy: a major cause of muscle weakness and paralysis. Lancet Neurol 2011;10:931-41 CrossRef

20. Shepherd S, Batra A, Lerner DP. Review of critical illness myopathy and neuropathy. Neurohospitalist 2017;7:41-48 CrossRef Medline

21. Gibson SB, Majersik JJ, Smith AG, et al. Three cases of acute myositis in adults following influenza-like illness during the H1N1 pandemic. J Neurosci Rural Pract 2013;4:51-54 CrossRef Medline

22. Dalakas MC, Pezeshkpour GH, Gravell M, et al. Poly-myositis associated with AIDS retrovirus. JAMA 1986;256:2381-83 CrossRef

23. Dalakas MC, Pezeshkpour GH. Neuromuscular diseases associated with human immunodeficiency virus infection. Ann Neurol 1988; 23:S38-S48 CrossRef

24. Villanova M, Caudai C, Sabatelli P, et al. Hepatitis C virus infection and myositis: a polymerase chain reaction study. Acta Neuropathol 2000;99:271-76 CrossRef Medline

25. Alsaad KO, Hajeer AH, Al Balwi M, et al. Histopathology of Middle East respiratory syndrome coronovirus (MERS-CoV) infectionclinicopathological and ultrastructural study. Histopathology 2018; 72:516-24 CrossRef Medline 